\title{
Avaliação físico-química e bioativa da polpa e geleia produzida a partir do fruto de Theobroma grandiflorum Schum (cupuaçu)
}

\section{Physical-chemical and bioactive evaluation of pulp and jelly produced from Theobroma grandiflorum schum (cupuaçu) fruit}

\author{
Ana Gabriela Vogado Couto ${ }^{1 *}$, Anderson Barriga Dos Santos ${ }^{2}$, Ziane da Conceição das \\ Mercês ${ }^{3}$, Anne do Socorro Santos da Silva ${ }^{4}$ \\ ${ }^{1}$ Bacharel em Nutrição, Acadêmica do curso de Nutrição, Faculdade Estácio Macapá. Macapá, AP. Brasil. https://orcid.org/0000-0001-8248- \\ 0631 E-mail: gabivcouto@yahoo.com.br*Autor para correspondência \\ ${ }^{2}$ Bacharel em Nutrição, Acadêmico do curso de Nutrição, Faculdade Estácio Macapá. Macapá, AP. Brasil. https://orcid.org/0000-0002- \\ 2334-803X E-mail: andersonsntos@gmail.com \\ ${ }^{3}$ Nutricionista Especialista em Docência na Educação Superior. Macapá, AP. Brasil. https://orcid.org/0000-0003-0646-2022 E-mail: \\ zianemerces@gmail.com \\ ${ }^{4}$ Nutricionista Professora curso de Nutrição da Faculdade Estácio de Macapá, pesquisadora do Instituto de Pesquisas Cientificas e \\ Tecnológicos do Estado do Amapá. Macapá, AP. Brasil. https://orcid.org/0000-0001-5524-8095 E-mail: annedosocorro@hotmail.com
}

\author{
Palavras-chave \\ Frutos Amazônicos \\ Composição centesimal \\ Ação Antioxidante
}

\begin{abstract}
Entre os frutos tropicais nativos da Amazônia, o cupuaçu é a fruta que apresenta uma grande importância para o extrativismo fomentando a economia nacional. Dentre os frutos tropicais nativos da Amazônia, o cupuaçu é o que reúne as melhores condições de aproveitamento industrial. Objetivou-se estudar a ação antioxidante na geleia produzida a partir do mesocarpo de Theobroma grandiflorum Schum, com intuito de avaliar a composição centesimal do fruto e da geleia. A metodologia empregada para a produção da pesquisa foi separada em tópicos, em que fazem descrição da matéria prima, infraestrutura, deposito no herbário, fluxograma, análises físico-químico e centesimal juntamente com os aspectos éticos legais. Resultados: Utilizando a polpa e a geleia de cupuaçu produzidos exclusivamente para essa pesquisa, com propósito de descrever as características físico-químicos e bioativos expressas na composição dos produtos derivados do Theobroma grandiflorum, obteve-se dados para teor de cinzas de 3,70 \% para polpa e 1,54\% para geleia, flavonoides foi 20,5 mg. $100 \mathrm{~g}^{-1}$ para polpa e de 2,86 mg.100 g $\mathrm{g}^{-1}$ para a geleia, entre outros resultados. Conclusão: Os valores correspondentes ao teor de cinzas, flavonóides, antocianinas e análises microbiológicos demonstram que tanto a fruta quanto os produtos derivados do cupuaçu são benéficos a saúde humana.
\end{abstract}

\section{Keywords}

Amazonian Fruits Centesimal composition Antioxidant action

\begin{abstract}
Among the tropical fruits native to the Amazon, cupuaçu is the fruit that is of great importance for extractivism, promoting the national economy. Among the tropical fruits native to the Amazon, cupuaçu is the one that brings together the best conditions for industrial use. The objective was to study the antioxidant action in the jelly produced from Theobroma grandiflorum Schum mesocarp, in order to evaluate the proximate composition of the fruit and jelly. The methodology used for the production of the research was separated into topics, in which they describe the raw material, infrastructure, deposit in the herbarium, flowchart, physical-chemical and centesimal analyzes together with the legal ethical aspects. Results: Using cupuaçu pulp and jelly produced exclusively for this research, with the purpose of describing the physical-chemical and bioactive characteristics expressed in the composition of products derived from Theobroma grandiflorum, data for ash content of $3.70 \%$ were obtained for pulp and 1.54\% for jelly, flavonoids was $20.5 \mathrm{mg} .100 \mathrm{~g}-1$ for pulp and $2.86 \mathrm{mg} .100 \mathrm{~g}^{-1}$ for jelly, among other results. Conclusion: The values corresponding to the content of ash, flavonoids, anthocyanins and microbiological analyzes show that both fruit and products derived from cupuaçu are beneficial to human health.
\end{abstract}


relata que o cupuaçu também é conhecido por cupu (do estado do Pará ao Acre); pupu, puaçu (Estado do Maranhão até a Bahia); cacau cupuaçu (Bahia); cupuazur (região de Iquitos no Peru), bacau (Colômbia), cacau blanco, pastate (México, Costa Rica, Panamá); patashte, cupuassu (Inglaterra); patas (Equador); lupu (Suriname). O nome cupuaçu vem da língua Tupi (Kupu = que parece com o cacau + uasu $=$ grande).

O Cupuaçuzeiro é uma das plantas frutíferas de maior importância para a Amazônia, principalmente devido a sua participação na composição dos sistemas de produção, cultivados e extrativos, além da grande aceitação e consumo da polpa de seus frutos (ALVES, 2013. Apud. SANTOS, 2008). Dentre os frutos tropicais nativos da Amazônia, o cupuaçu é o que reúne as melhores condições de aproveitamento industrial.

Carvalho (2015), descreve que a região Amazônica é o local onde a frutífera encontra as melhores condições edafoclimáticas para o seu desenvolvimento. Porém, temperatura e umidade relativa do ar elevadas, e condições de cultivo, colheita e armazenamento inadequados acabam desencadeando e acelerando o processo de deterioração pós-colheita.

Portanto o presente trabalho propõe em seu objetivo estudar a ação antioxidante na geleia produzida a partir do mesocarpo de Theobroma Grandiflorum Schum, com intuito de avaliar a composição centesimal do fruto e da geleia.

\section{MATERIAL E MÉTODOS}

\section{Matéria prima}

Os frutos foram adquiridos comercialmente na feira do produtor do Buritizal no município de Macapá - AP, entre os meses de março a junho de 2019, que são o período que corresponde à safra do cupuaçu.

\section{Infraestrutura}

A pesquisa foi realizada na Faculdade Estácio de Macapá, no laboratório de bromatologia e tecnologia dos alimentos. O outro laboratório utilizado para seguimento do estudo foi o laboratório de bromatologia e tecnologia dos alimentos do Instituto de Pesquisa Científicas e Tecnológicas do Estado do Amapá (IEPA), no Núcleo de ciência e tecnologia de Alimentos.

\section{Depósito no herbário}

Antecedendo a pesquisa, foi depositado no herbário do
Instituto de Pesquisa Científicas e Tecnológicas do Estado do Amapá (IEPA), Herbário Amapaense (HAMAB), uma exsicata do Theobroma Grandiflorum Schum (cupuaçu) utilizado na produção da geleia, para comprovação, definição e classificação do gênero e espécie utilizados pelo estudo. $O$ responsável pela identificação da espécie herborizada foi o Me. Tonny David Santiago Medeiros - Curador do HAMAB, após o analise obteve-se o Parecer № 16/2019 HAMAB/NUBIO/IEPA. A coleta do fruto foi realizada na cidade de Macapá - AP, Jardim Felicidade, Avenida Geribá de Almeida Azevedo Costa. Coord.: Lat. 005`01.5” N Long.: 51을.2” W.

\section{Seleção e Limpeza}

Os frutos do cupuaçu foram selecionados criteriosamente, um a um, considerando os aspectos de maturação, qualidade dos frutos, defeitos para possível descarte. Em seguida, foram higienizados, a primeira lavagem apenas com água para a retirada de sujidades, seguindo para segunda lavagem submersos em água clorada (200 ppm de cloro livre) por 30 minutos, e a terceira lavagem em água potável para retirada do resíduo de cloro como apresentados na (Figura 1).

\section{Despolpamento e Armazenamento}

Os frutos foram quebrados e retirados da casca, armazenados em recipientes para posteriormente serem separados o mesocarpo dos caroços. O despolpamento foi realizado manualmente com auxílio de tesoura de aço inox, devidamente higienizada e esterilizada. O mesocarpo foi submetido à cocção, utilizando um tacho de aço inoxidável por processo manual em fogão industrial, em temperatura a 90 ํ. Após sua cocção o produto final da polpa do cupuaçu foi resfriado até 60 ㅇ. . Os recipientes que receberam a geleia foram previamente sanitizados.

O procedimento do envase foi realizado por meio do enchimento quente, em frasco de vidro esterilizado. Após o enchimento, colocou-se a tampa e inverteu-se o frasco de ponta cabeça, permanecendo nessa posição por um período entre 10 a 15 minutos, seguindo para o resfriamento total. 0 resfriamento foi realizado por imersão dos frascos em água morna a 70 ㄷ. Sendo substituída de modo gradual por água fria, para não trincar os frascos de vidro (Figura 1).

A geleia pode ser estocada à temperatura ambiente inferior a 30 oc , em local seco, fresco e arejado, apresentando prazo de prateleira por até 12 meses segundo Souza (2007). 
Figura 1. Imagens desde a pesagem da polpa até produto final geleia.
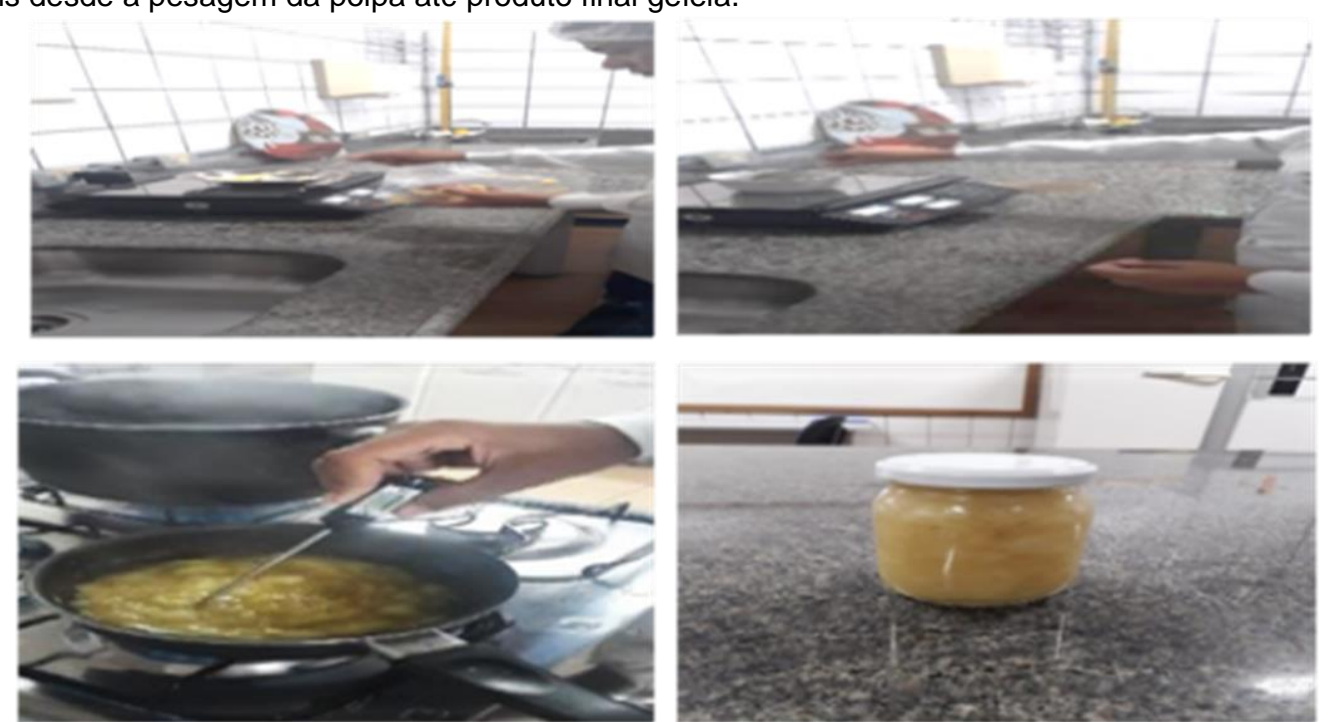

Em (A) e (B) primeira etapa da pesagem da polpa do cupuaçu, em (C) verificação da temperatura por termômetro para alimentos, e em (D) geleia de cupuaçu envasada, e pronto para consumo. Fonte: Dados da pesquisa.

Figura 2. Fluxograma do processo de despolpamento do cupuaçu (Theobroma grandiflorum Schum)

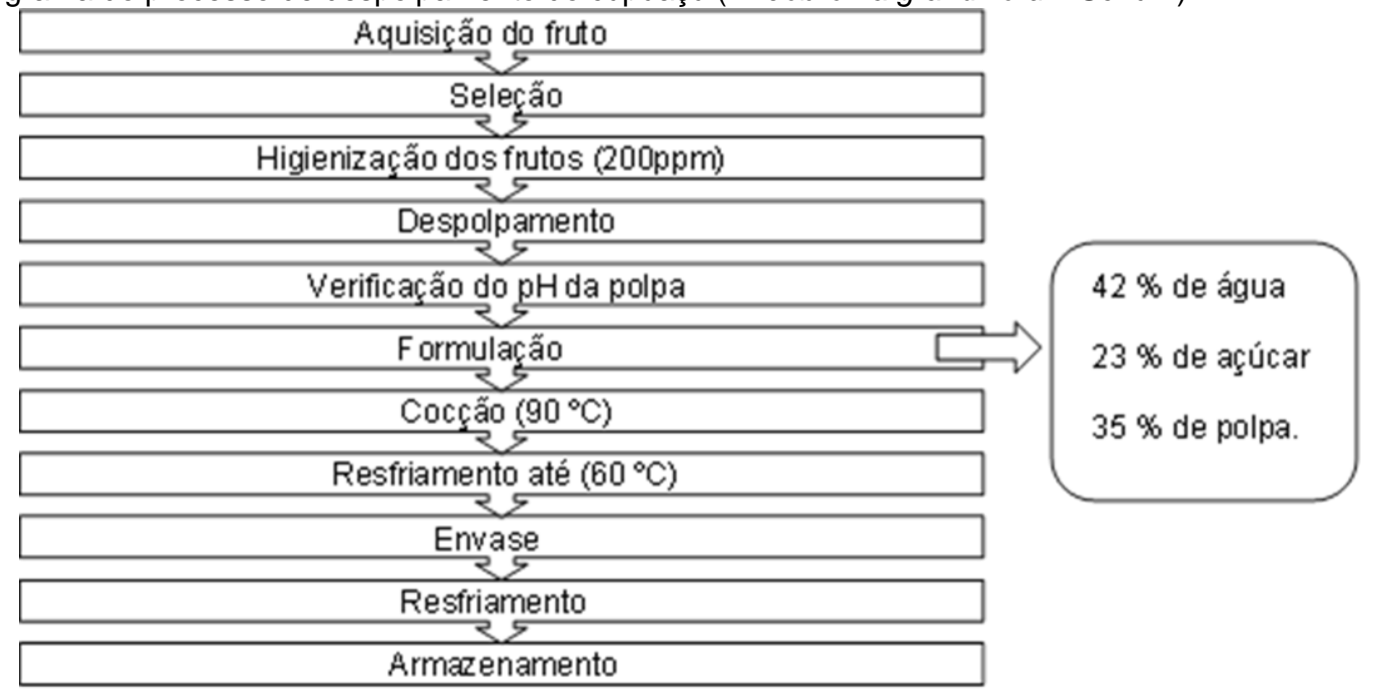

Fonte: Dados da pesquisa.

\section{Formulação da geleia}

A produção da geleia foi realizada com a seguinte formulação, $150 \mathrm{ml}$ de água (42\%), $100 \mathrm{~g}$ de açúcar (23\%), $200 \mathrm{~g}$ de polpa de cupuaçu (35\%).

\section{Caracterização físico-químicas}

A caracterização físico-química das polpas desidratadas e das geleias foi realizada através da aplicação de metodologias segundo Association of Official Analytical Chemists (AOAC, 2016), sendo: umidade, cinzas, proteínas, pectina, acidez, grau Brix. Todas as análises da caracterização físico-química foram realizadas em triplicata.

\section{Umidade}

As amostras foram pesadas em Becker de vidro contendo 2,0 g de cada amostra, a secagem foi realizada em estufa (Medicate ${ }^{\oplus}$, Mod. MD 1.2), à temperatura de 105 oC por aproximadamente 1 horas, de acordo com a metodologia $\mathrm{n}$ ㅇ 925.09 da AOAC (2016). Após a secagem, as amostras foram retiradas da estufa e colocadas para esfriar no dissecador (Schott, estrutura de Policarbonato Transparente $250 \mathrm{~mm}$ ), por um tempo mínimo de $30 \mathrm{~min}$.

\section{Cinzas}

As amostras foram pesadas em cadinhos de porcelana contendo 2,0 g de cada amostra, e levados para calcinação a 550 으, de acordo com o método gravimétrico $n^{\circ} 923.03$ da AOAC (2016), com permanência da amostra na mufla até a 
queima de toda a matéria orgânica. O resultado foi expresso em $\mathrm{g} / 100 \mathrm{~g}$ de cinzas na amostra.

\section{Proteínas}

Após a digestão da amostra com ácido sulfúrico e a mistura catalítica, foi realizada a destilação em ácido bórico a $4 \%$ após neutralização com hidróxido de sódio a $40 \%$. Em seguida foi feita a titulação com ácido clorídrico a $0,1 \mathrm{~N}$, de acordo com o método de micro Kjeldahl $n^{\circ} 920.87$ da AOAC (2016). Para a realização do cálculo utilizou-se 5,75 como fator de conversão de $\mathrm{N}$ em proteína, expresso em g/100 g de proteína na amostra.

\section{pH}

Para a determinação do $\mathrm{pH}$ foi feita pelo método potenciométrico, utilizando-se $5 \mathrm{~g}$ de amostras diluída em 50 $\mathrm{mL}$ de água. A medida foi feita em um pHmetro de bancada (Hanna Instruments, Mod. HI3222, precisão 0,01), devidamente calibrado com soluções-tampão de pH 4,0 e 7,0 (Instituto Adolfo Lutz, 2008).

\section{Brix}

Para a análise de grau Brix foi utilizado refratômetro de escala de 0 a 32, calibrado com água (IAL, 2008).

\section{Lipídeos}

A determinação da fração lipídica foi realizada pelo método de Bligh-Dyer conforme descrito por Brum et al. (2009) as amostras foram pesadas aproximadamente $10 \mathrm{~g}$ de cada amostra. Em um Erlenmeyer de $250 \mathrm{~mL}$ foram adicionados $50 \mathrm{~mL}$ de metanol, $25 \mathrm{~mL}$ de clorofórmio e 10 $\mathrm{mL}$ de água.

\section{Determinação dos compostos bioativos}

Os compostos bioativos podem estar presentes em diversos tipos de alimentos, como nas ervas, frutas, grãos etc. Eles são encontrados sob duas formas, os que agem promovendo a saúde, chamados nutracêuticas e os que podem causar danos à mesma, os tóxicos. Sendo assim, se um alimento que possui compostos bioativos contém mais substâncias nutracêuticas do que tóxicas, ele pode ser considerado um agente protetor contra doenças crônicas (CASTELUCCI, 2015).

\section{Antocianinas}

O teor de antocianinas foi realizado conforme descrito por IAL (2008). Determinado em mg 100 g-1, e convertido para $\mu \mathrm{g} 100 \mathrm{~g}-1$.

Para determinação da antocianina multiplica-se a amostra por 1.000 para obter em $\mu \mathrm{g} 100 \mathrm{~g}-1$. Em alimentos usa-se $\mu \mathrm{g} 100 \mathrm{~g}$-1.

\section{Flavonóides}

O conteúdo de flavonoides foi determinado conforme descrito por Kuskoski et al. (2006).

\section{Analise microbiológica}

Os testes microbiológicos empregados foram realizados conforme descrito por Silva; Junqueira e Silveira (2001).

\section{Análise estatística}

As análises foram conduzidas em triplicata e os dados expressos como média \pm desvio padrão (DP), utilizando o programa Microsoft Excel versão 2019.

\section{RESULTADOS E DISCUSSÃO}

Na Tabela 1 abaixo estão apresentados os resultados dos parâmetros físico-químicos e bioativos da polpa e geleia do mesocarpo de cupuaçu.

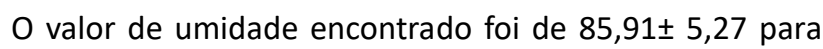
polpa de cupuaçu utilizada na produção da geleia. A umidade em alimentos está diretamente relacionada com a estabilidade e qualidade do produto (MAMANI-LINARES; GALLO, 2014). Valores semelhantes foram encontrados nos estudos de Nascimento et al. (2019), correspondendo a $86,73 \pm 0,4$. Dados superiores foram descritos nos estudos de Canuto et al. (2010). Correspondendo a 89,2 \% a partir da polpa in natura de cupuaçu.

Os resultados obtidos pelo estudo para a umidade da geleia produzida a partir do mesocarpo do cupuaçu apresentaram pequenas variações entre as três amostras, com resultados de $63,15 \%$ a $64,24 \%$, apresentando resultado final para umidade da geleia de $37,89 \%$, esse quantitativo apresenta-se dentro das normas estabelecidas pela Resolução CNNPA 12 de 1978 (BRASIL, 1978) que descreve o mínimo e o máximo de $35 \%$ e 38 respectivamente. Os valores apresentados pelo estudo foram superiores aos dados apresentados pela pesquisa de Santos et al. (2014) que obtiveram valor de $35,54 \%$ para geleia produzida da 
Tabela 1. Resultados das análises físico-química da polpa e da geleia produzida a partir do mesocarpo do cupuaçu.

\begin{tabular}{cccc}
\hline Análise & $\begin{array}{c}\text { *Polpa do } \\
\text { cupuaçu }\end{array}$ & $\begin{array}{c}\text { *Geleia a partir } \\
\text { do mesocarpo }\end{array}$ & $\begin{array}{c}\text { Referência } \\
\text { Padrão p/ } \\
\text { geleia }\end{array}$ \\
\hline Umidade (\%) & $85,91 \pm 5,27$ & $63,82 \pm 0,61$ & 30,47 \\
\hline Cinzas (\%) & $3,70 \pm 0,38$ & $1,54 \pm 0,00$ & 0,23 \\
\hline Lipídeos (\%) & $0,39 \pm 0,11$ & $11,70 \pm 0,73$ & 0,07 \\
\hline Proteína (\%) & $2,61 \pm 0,11$ & $1,65 \pm 0,08$ & 0,37 \\
\hline $\begin{array}{c}\text { Antocianina (mg } \\
100 \mathrm{~g}^{-1} \text { ) }\end{array}$ & $2,58 \pm 0,80$ & $1,71 \pm 0,79$ & - \\
\hline Flavonoides (mg & & & - \\
$\left.100 \mathrm{~g}^{-1}\right)$ & $20,5 \pm 3,0$ & $2,86 \pm 0,78$ & \\
\hline
\end{tabular}

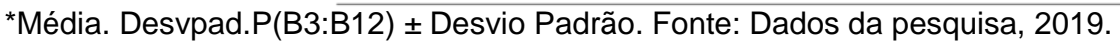

fruta abacaxi. A diminuição da atividade de água da fruta contribui para a conservação, quando um material biológico é exposto a uma certa umidade, ele perde ou ganha água para ajustar sua própria umidade a uma condição de equilíbrio com o ambiente. As informações acerca da umidade de equilíbrio são importantes no processamento e armazenamento de alimentos (FOPPA et al., 2009).

O conteúdo de cinzas das amostras da polpa e da geleia de cupuaçu foram respectivamente de $(3,70 \%)$ e $(1,54 \%)$. Segundo Krumreich et al. (2013), o teor de cinzas em alimentos refere-se ao resíduo inorgânico, ou resíduo mineral fixo (sódio, potássio, magnésio, cálcio, ferro, fósforo, cobre, cloreto, alumínio, zinco, manganês e outros compostos minerais), nutrientes essenciais que devem ser consumidos diariamente para a manutenção da homeostase corporal. Estes nutrientes são importantes pois, atuam na formação de ossos e dentes, transmissão neural, contração muscular, equilíbrio ácido-base, cofatores enzimáticos, controle do potencial de membranas e tônus vascular (BECKER et al., 2018). Os resultados para teores de cinzas apresentados pelos estudos de Martins e Martim (2020); Alves (2013), foram inferiores ao deste estudo, com resultados de $1,0 \%$ e $1,36 \%$ respectivamente, compreendese que fatore como solo e clima interferem diretamente nos resultados.

Os dados obtidos pela pesquisa para teores lipídicos para polpa e geleia de cupuaçu foram de $0,13 \%$ e $5,05 \%$ respectivamente. Dados superiores $(0,41 \%)$ para polpa de cupuaçu foram descritos no estudo de Nascimento et al. (2019).

Os resultados expressos nesta pesquisa referentes a proteína da polpa e geleia de cupuaçu foram de $2,61 \%$ e $1,65 \%$ respectivamente, a diminuição da proteína da polpa do cupuaçu ao transforma-la em geleia, pode ser explicado pela utilização de altas temperaturas durante o procedimento de cocção (BENOTI; RAMOS, 2015). Segundo dados apresentados por Alves (2013), em que apresenta resultado de 3,45 \% para amostra da polpa do cupuaçu, superior ao descrito neste estudo.

O papel dos flavonoides e demais compostos deste grupo são descritos como responsáveis em parte pelos efeitos protetores de uma dieta rica em frutas e vegetais e cada vez mais se tornam importantes as pesquisas dos mesmos e a nutrição humana (ARAÚJO, 2017). Os flavonoides são pigmentos naturais amplamente distribuídos no reino vegetal. Protegem o organismo do dano produzido por agentes oxidantes como os raios ultravioletas, poluição ambiental, substâncias químicas presentes nos alimentos, estresses, dentre outros (GADELHA, 2016). Resultados obtidos nas analise para flavonoides foi de $20,5 \mathrm{mg} .100 \mathrm{~g}^{-1}$ para a polpa de cupuaçu, de 2,86 mg.100 g-1 para a geleia. Valores superiores ao desta pesquisa foram descritos no estudo de Meneses et al. (2016), em que os resultados para flavonoides (mg. catequina100g-1) foram de 11,75 \pm 11,31 para polpa de goiaba. No estudo de Gadelha (2016), os resultados descritos para flavonoides foram inferiores à deste estudo, apresentando valores de $0,38 \mathrm{mg} \cdot 100 \mathrm{~g}^{-1}$ para polpas de abacaxi e $4,85 \mathrm{mg} \cdot 100 \mathrm{~g}^{-1}$ tamarindo. Os teores de flavonoides em frutas podem variar até $100 \%$ entre um semestre e outro, sendo estes influenciados por fatores extrínsecos tais como condições climáticas, época de plantio, colheita e grau de maturidade dos frutos; o que valoriza os resíduos das frutas tropicais (ARAÚJO, 2017).

Os valores obtidos para antocianinas, foram descritos através das análises nas amostras da polpa e da geleia de cupuaçu, em que os resultados foram de $2,58 \mathrm{mg} .100 \mathrm{~g}^{-1}$ para 
polpa de cupuaçu, e de $1,71 \mathrm{mg} \cdot 100 \mathrm{~g}^{-1}$ para a geleia. Valores inferiores foram descritos no estudo de Fonseca (2014), em que apresentou o teor de antocianina para a polpa de abacaxi de $0,21 \mathrm{mg}$. $100 \mathrm{~g}^{-1}$. No estudo de Gadelha (2016), as quantidades apresentadas variaram entre $0,17 \mathrm{mg} \cdot 100 \mathrm{~g}^{-1}$ para a polpa de maracujá e $4,95 \mathrm{mg} \cdot 100 \mathrm{~g}^{-1}$ para a polpa de acerola. Os valores baixos para antocianinas sugerem que as amostras analisadas sofreram degradação, podendo ter sido ocasionado pela mudança de temperatura, exposição a luz ou por enzimas, já que são pigmentos muito instáveis (GADELHA, 2016).

Os sólidos solúveis da polpa de cupuaçu foi 9,10 Brix, os resultados obtidos para sólidos solúveis da geleia foi 33,1 oBrix. Valores superiores foram descritos no estudo de Costa et al. (2018), em que apresenta 12,1 -Brix para polpa de cupuaçu. No estudo realizado por Silva (2014) foi encontrado resultado superior de $68{ }^{\circ} \mathrm{Brix}$ para geleia. Os resultados apresentados pelo estudo estão de acordo com a Instrução Normativa № 01, de 7 de janeiro de 2000, as características físico-químicas da polpa de cupuaçu devem ter no mínimo 9,0 Brix (BRASIL, 2000).

Os valores obtidos para $\mathrm{pH}$ na polpa e geleia de cupuaçu foi de 3,75 e 3,80 respectivamente. Valores inferiores foram descritos por Costa et al. (2018), com resultado de 3,1 para polpa de cupuaçu. No estudo de Gadelha (2016), as médias de $\mathrm{pH}$ das polpas das frutas variaram de 2,99 (polpa de tamarindo) a 4,15 (polpa de abacaxi), classificando-as como ácidas, característica importante que inibe o desenvolvimento de microrganismos bacterianos. Esses resultados para a polpa avaliada estão de acordo com a legislação vigente, cujo o valor mínimo para $\mathrm{pH}$ da polpa de cupuaçu é de 2,6 (BRASIL, 2000).

Os resultados das análises microbiológicas realizadas na geleia de cupuaçu obedeceram ao padrão da legislação brasileira vigente para polpas de frutas no tocante a Coliformes $45^{\circ} \mathrm{C}$ e Salmonella sp conforme apresentados na tabela 2.

Esses resultados mostraram boas condições higiênicosanitárias no processamento da geleia de cupuaçu indicando a correta aplicação das boas práticas de fabricação (BPF). Resultados semelhantes foram descritos no estudo de Goncalves et al. (2014), expressando dados para coliformes totais a $450 \mathrm{C}$ de $(<3 \mathrm{NMP} / \mathrm{g})$ e ausência para salmonela $\mathrm{sp}$. Na pesquisa de Martins e Martim (2020), em que as análise microbiológicas da geleia do cupuaçu não apresentaram microorganismos contaminantes. No estudo de Oliveira et al. (2016), não apresentaram coliformes termotolente para geleia de laranja enriquecida com aveia.

\section{CONCLUSÕES}

Utilizando a polpa e a geleia de cupuaçu produzidos exclusivamente para essa pesquisa, com propósito de descrever as características físico-químicos e bioativos expressas na composição dos produtos derivados do Theobroma grandiflorum, obteve-se resultados expressivos sobre esse fruto genuinamente amazônico. Os valores correspondentes ao teor de cinzas, flavonóides, antocianinas e análises microbiológicos demonstram que tanto a fruta quanto os produtos derivados do cupuaçu são benéficos a saúde.

\section{AGRADECIMENTOS}

Ao Instituto de Pesquisas Científicas e Tecnológicas do Estado do Amapá (IEPA) e Faculdade Estácio de Macapá, pelo apoio estrutural, que foram essenciais para o desenvolvimento da pesquisa.

\section{REFERÊNCIAS}

ALVES, R. M.; FERNANDES, J. R. Q. A Cultivar de

Tabela 2. Parâmetros microbiológicos para polpa e geleia do mesocarpo de cupuaçu.

\begin{tabular}{cccc}
\hline Análise & $\begin{array}{c}\text { Polpa de } \\
\text { cupuaçu }\end{array}$ & $\begin{array}{c}\text { Geleia de } \\
\text { cupuaçu }\end{array}$ & $\begin{array}{c}\text { *Limite } \\
\text { estabelecido }\end{array}$ \\
\hline $\begin{array}{c}\text { Coliformes } \\
\text { totais a 45 } \mathbf{C}\end{array}$ & - & $<3$ & $10^{2} \mathrm{UFC}^{*}$ \\
$(\mathrm{NMP} / \mathrm{g})^{*}$ & & & \\
\hline Salmonela sp. & - & - & - \\
\hline
\end{tabular}

*Estimado contagem inferior a 25 colônias e superior a 250 colônias. (-) ausência em 25 g. *NMP= Número mais provável. *UFC= Unidade formadoras de colônias. Fonte: Dados da pesquisa, 2019. 
Cupuaçuzeiro BRS Carimbó. XXII Congresso Brasileiro de Fruticultura. Bento Gonçalves-RS, 2012.

ALVES, D.P. Determinação de Características Físico-químicas de Polpas de Cupuaçu (Theobroma Grandiflorum Schum) Congeladas Comercializadas em Ariquemes, Rondônia, Brasil. Ariquemes -RO, 2013.

ALVES, R. M.; FILGUEIRAS, G. C.; HOMMA, A.K.O. Aspectos socioeconômicos do Cupuaçuzeiro na Amazônia: do extrativismo a domesticação. In: SANTANA, A. C. (ed.). Mercado, cadeias produtivas e desenvolvimento rural na Amazônia. 1.ed. Belém, PA: UFRA, 2014. p.197-223.

AOAC. Association Of Official Analytical Chemists Official methods of analysis of the Association of Official Analytical Chemists: edited Dr. George W. Latimer, Jr. 20a ed. Washington, 2016.

ARAÚJO, Ingrid Maria Cardoso de et al. Caracterização bioativa de resíduos de frutas tropicais. 2017. Trabalho de Conclusão de Curso. Universidade Federal do Rio Grande do Norte.

BANCO DA AMAZÔNIA-S/A-GEROP-COASC, Relatórios, 2012.

BECKER, M. M.; MANDAJI, C. M.; CATANANTE, G.; MARTY, J. L.; NUNES, G. S. Mineral and bromatological assessment and determination of the antioxidant capacity and bioactive compounds in native Amazon fruits. Brazilian Journal of Food Technology, Campinas, v. 21, p. 1-9, 2018.

BINOTI, M. L.; RAMOS, A. M. Conservação de alimentos: uma visão mais saudável. HU Revista, v. 41, n. 3 e 4, 2015.

BRASIL, Ministério da Agricultura Pecuária e do Abastecimento (MAPA). Instrução Normativa № 01, de 07/ 01/ 2000. Disponível em: < www2.agricultura.rs.gov.br/uploads/126989581629.03_e nol_in_1_00_mapa.doc $+\& c d=2 \& h l=p t-R \& c t=c \mid n k \& g l=b r>$ Acesso em 23/1111/2019

BRASIL, Agência Nacional de Vigilância Sanitária (ANVISA). Resolução - RDC no 12, de 2 de janeiro de 2001 Regulamento Técnico Sobre os Padrões Microbiológicos Para Alimentos. Disponível em:<http://portal.anvisa.gov.br/wps/wcm/connect/a47b ab8047458b909541d53fbc4c6735/RDC_12_2001.pdf?M OD=AJPERES> Acesso em: 26/11/2019.

BRASIL. Agência Nacional de Vigilância Sanitária. ANVISA. Resolução 12, de 24 de julho de 1978. Aprova Normas Técnicas Especiais, do Estado de São Paulo, revistas pela CNNPA, relativas a alimentos (e bebidas), para efeito em todo território brasileiro. DOU, Brasília, 24 de julho de 1978. Disponível em: http://portal.anvisa.gov.br/wps/wcm/connect/e57b7380 474588a39266d63fbc4c6735/RESOLUCAO_12_1978. pdf?MOD=AJPERES. Acesso em: 26/11/2019.
BRUM, A. A. S.; ARRUDA, L. F. de; REGITANO-D'ARCE, M. A. B. Métodos de extração e qualidade da fração lipídica de matérias-primas de origem vegetal e animal. Quim. Nova, vol. 32, no. 4, 849-854, 2009.

CAETANO, P. K.; DAIUTO, E. R.; VIEITES, R. L. Característica físico-química e sensorial de geleia elaborada com polpa e suco de acerola. Braz. J. Food Technol, Campinas, v. 15, n. 3, p. 191-197, Jul. /set. 2012

CHAAR, M. J. A composição do cupuaçu (Theobroma grandiflorum Schum) e conservação de seu néctar por meio físicos e químicos. 1980. 87p. Dissertação (Mestrado em ciências de Alimentos) - Universidade Rural do Rio de Janeiro, Rio de Janeiro, 1980.

CANUTO, G. A.; XAVIER, A. A. O.; NEVES, L. C.; BENASSI, M. T. Caracterização físico química de polpas de frutos da Amazônia e sua correlação com a atividade anti-radical livre. Revista Brasileira de Fruticultura, v. 32, p. 11961205, 2010.

CARVALHO, A. V. Extração, concentração e caracterização físico-química e funcional das proteínas de semente de cupuaçu (Theobroma grandiflorum Schum). Tese apresentada a Faculdade de Engenharia de Alimentos da Universidade Estadual de Campinas. -Campinas -SP, 2004.

CARVALHO, A.V.; GARCÍA, N.H.P.; FARFÁN, J.A. Proteínas da semente de cupuaçu e alterações devidas à fermentação e à torração. Ciência e Tecnologia de Alimentos. Campinas, v. 28, n.4, p.986-993, 2008.

CARVALHO, C.A.C.; ÁlVARES, V.S.; CUNHA, C.R.; LIMA, A.A.; MORENO, A.L.; MACIEL, V.T. Efeito do pré-resfriamento de frutos de cupuaçu na aceitação sensorial do néctar. Revista Agro@mbiente On-line, v. 9, n. 1, p. 91-95, janeiro-março, 2015.

CASTELUCCI, A. C. L. Avaliação da estabilidade dos compostos bioativos de polpas de frutas nativas submetidas ao processo de irradiação/Tese de doutorado. Piracicaba, 2015. 133 p.

CLEMENT, C. R.; ARAÙJO, M. C.; GEO COPPENS D'EECKENBRUGGE, Pereira, A. A., and PICANÇORODRIGUES, D. Origin and Domestication of Native Amazonian Crops. Diversity, ed. 2, p. 72-106; 2010.

COSTA, R. A.; LIMA, D. E. S.; SOUSA, E. P.; LEMOS, D. M.; GOMES, F. P.; NASCIMENTO, N. M. Caracterização físicoquímica de geleia de Cupuaçu e açaí (blend) oriundas da região Amazônica.60 Simpósio de segurança alimentar. Gramado, RGS, 2018.

Deparment of Agriculture. USDA. Agricultural Research Service: USDA Nutrient Database for Standard Reference; 2001.

EMBRAPA. Cupuaçuzeiro. Disponível pelo endereço eletrônico 
em<http:www.cpatu.embrapa.br/fruteiras.htm>. Acesso em 19/02/2019.

FONSECA, A. V. V. da. Perfil sensorial, aceitação e caracterização em compostos bioativos de néctares mistos de frutas tropicais/Tese (Doutorado) - Curso de Ciência e Tecnologia de Alimentos. Coordenação do Curso de Pós-graduação em Ciência e Tecnologia de Alimentos, Universidade Federal do Ceará, Fortaleza, 2014.

FOPPA, T.; TSUZUKI, M.; SANTOS, C. E. S. Caracterização físico-química da geleia de pera elaborada através de duas cultivares diferentes: pêra d'água (Pyrus Communis I.) E Housui (Pyrus pyrifolia nakai). Revista Brasileira de Produtos Agroindustriais, Campina Grande, v.11, n.1, p.21-25, 2009.

FREIRE, M. T. A et al. Caracterização físico-química, microbiológica e sensorial de polpa de cupuaçu congelada (Theobroma grandiflorum Schum) Rev. Braz. J. Food Technol. v. 12, n. 1, p. 09-16, 2009.

GADELHA, M. R.A. Desenvolvimento de blends com frutos tropicais à base de tamarindo. Pombal, 2016.

GONÇALVES, M. V. V. A.; DA SILVA, J. P. L.; ROSENTHAL, A.; FURTADO, A. A. L.; CALADO, V. M. DE A. Incidência de fungos termorresistentes e propriedades microbiológicas da polpa de cupuaçu congelada (Theobroma grandiflorum Schum). Biológicas \& Saúde, v. 4, n. 14, 18 dez. 2014.

GONÇALVES, M.V.V.A. Segurança Microbiológica de Polpa de

Cupuaçu Processada Termicamente. Programa pósgraduação em Tecnologia de Processos Químicos e Bioquímicos da Escola de Química da Universidade Federal do Rio de Janeiro. Rio de Janeiro- RJ, 2014.

Instituto Adolfo Lutz (São Paulo). Métodos físico-químicos para análise de alimentos /coordenadores Odair Zenebon, Neus Sadocco Pascuet e Paulo Tiglea -- São Paulo: Instituto Adolfo Lutz, 2008 p. 1020

KHODDAMI, A.; WILKES, M. A.; ROBERTS, T. H. Techniques for analysis of plant phenolic compounds. Molecules, v. 18, n. 2, p. 2328-75, 2013.

KRUMREICH, F. D.; SOUSA, C. T.; CORRÊA, A. P. A.; KROLOW, A. C. R.; ZAMBIAZI, R. C. Teor de cinzas em acessos de abóboras (cucurbita máxima I.) do Rio Grande do Sul. VIII simpósio de alimentos. v.8. 2013 p. 1/4

LIMA, M.F.; SILVA, D.C.; VELLEZ, H.A.V.; SANTANA, A.A. Influência do Mesocarpo de

Coco Babaçu na Formação de Biofilmes Aditivados com Compostos Antioxidantes - Cupuaçu (Theobroma Grandiflorum). XII Congresso Brasileiro de Engenharia Química em Iniciação Científica UFSCar - São Carlos - SP, 16 a 19 de Julho de 2017.

LÓPEZ, P.A.B. Avaliação da Cadeia Produtiva do Cupuaçu
(Theobroma Grandiflorum (Willd. Ex Spreng.) Schum) nos Municípios de Itacoatiara, Presidente Figueiredo e Manaus. Programa de Pós-Graduação em Agricultura no Trópico Úmido-Atu. Manaus- Amazonas, 2015.

MARTIN, L.G.P. Estudo da Secagem da Polpa de Cupuaçu por Atomização. Programa de Pós Graduação em Engenharia Agrícola da Faculdade de Engenharia Agrícola da Universidade Estadual de Campinas. Campinas, 2013.

MARTINS, M. N. N.; MARTIM, S. R. Geleia mista de tucumã e cupuaçu enriquecida com exocarpo de banana verde. Saber Científico, Porto Velho, v. 9, n. 1, p. 90-101, jan./jun. 2020.

MATTIETO, R. A. Estudo comparativo das transformações estruturais e físico-químico durante o processo fermentativo de amêndoas de cacau (Theobroma cacao L.) e cupuaçu (Theobroma grandiflorium Schum.). 2011. 164 f. Dissertação (Mestrado em Tecnologia de Alimentos) - Faculdade de Engenharia de Alimentos, Universidade Estadual de Campinas, Campinas. 2011.

MENEZES, P. E.; DORNELLES, L. L.; FOGAÇA, A. O.; BOLIGON, A. A.; ATHAYDE, M. L.; BERTAGNOLLI, S. M. M. Composição centesimal, compostos bioativos, atividade antioxidante e caracterização fenólica da polpa de goiaba. Disciplinarum Scientia| Saúde, v. 17, n. 2, p. 205217, 2016.

MUNDIM, S. M.; BUCHWEITZ, P. R.; VIEIRA, L.; MUNDIM, A. P. M.; LINS, N.; BRITO, C. R. N. Processamento de geleia de cupuaçu: uma alternativa econômica para o produtor rural do Amazonas. Higiene Alimentar - Vol. 27 - no 218/219 - março/abril de 2013.

NASCIMENTO, J. F.; ANTONIO JÚNIOR, C. S. S.; TOSTES, E. S. L.; SILVA, A. S. S. da. Avaliação físico-química de polpas de cupuaçu, Theobroma grandiflorum Schum, industriais e artesanais. PUBVET. v.13, n.3, a300, p.1-6, Mar., 2019.

NAZARÉ, R. F. R. de. Produtos Agroindustriais de Bacuri: Cupuaçu, graviola e açaí, desenvolvidos pela Embrapa Amazônia Oriental. Belém: Embrapa, 2000. (Documentos 41).

OLIVEIRA, K. A. M.; OLIVEIRA, G. V.; BATALINI, C.; ROSALEM, J. A.; RIBEIRO, L. S. Atividade antimicrobiana e quantificação de Flavonoides e Fenóis totais em diferentes extratos de Própolis. Semina: Ciências Biológicas e da Saúde. Londrina, v. 33, n. 2, p. 211222, jul./dez. 2012.

OLIVEIRA, C. F. D.; PINTO, E. G.; TOMÉ, A. C.; QUINTANA, R. C.; DIAS, B. F. Desenvolvimento e caracterização de geleia de laranja enriquecida com aveia. Revista de Agricultura Neotropical, Cassilândia-MS, v. 3, n. 3, p. 20-23, 2016.

OLIVEIRA, S. G. de. Avaliação do potencial de formação de peptídeos inibidores da enzima conversora da angiotensina I a partir de hidrolisados proteicos de 
amêndoas de cupuaçu fermentadas / Sabrina Grizzi de Oliveira. -93 p. Dissertação (Mestrado) - Faculdade de Ciências Farmacêuticas da Universidade de São Paulo. São Paulo, 2017.

PAGLARINI, C. S.; SILVA, F. S.; PORTO, A. G., SANTOS, P.; LEITE, A. L. M. P. Avaliação Físico-Química de Polpas de Frutas Congeladas Comercializadas na Região do Médio Norte Mato-grossense. Enciclopédia Biosfera, Goiânia, vol.7, N.13; p. 1391, 2011.

RIBEIRO, A. L. Z.; DEUS, J. C. S. de; MONTEIRO, B. P.; PEREIRA, D. S. Espécies botânicas de interesse socioeconômico e meliponícola nos municípios de Almeirim, Curuçá e Monte Alegre - Pará, Brasil. In: Seminário de Iniciação Científica da Embrapa Amazônia Oriental, 22., 2018, Belém, PA. Anais... Belém, PA: Embrapa Amazônia Oriental, 2018.

PUGLIESE, A. G. Compostos fenólicos do cupuaçu (Theobroma grandiflorum) e do cupulate: Composição e possíveis benefícios. 2010. (Mestrado na área de Bromatologia) - Universidade de São Paulo Faculdade de Ciências Farmacêuticas - Programa de Pós-Graduação em Ciência dos Alimentos, São Paulo, 2010.

ROGEZ, H.; BUXANT, R.; MIGNOLET, E.; SOUZA, J. N. S.; SILVA, E. M.; LARONDELLE, Y. Chemical composition of the pulp of three tipical Amazonian fruits: araça-boi (Eugenia stipitata), bacuri (Platonia insignis) and cupuaçu (Theobroma grandiflorum). European Journal of Research and Technology, n 218, p 380-384, 2004.

RUFINO, M. S. M.; ALVES, R. E.; BRITO, E. S.; PÉREZ-JIMÉNEZ, J.; SAURA-CALIXTO, F.; MANCINI-FILHO, J. Bioactive compounds and antioxidant capacities of 18 nontraditional tropical fruits from Brazil. Food Chemistry, v. 121, p. 996-1002, 2010.

SALGADO, H. L. C. Sobremesa láctea de cupuaçu (Theobroma grandiflorum Schum): desenvolvimento e estudo da vida de prateleira. Programa de PósGraduação em Cien. Tecn. dos alimentos. Belém- PA, 2010.

SANTOS, A.L.; PEZOA-GARCÍA, H. N.; AMAYA-FARFÁN, J. Qualidade nutricional das proteínas de cupuaçu e de cacau. Ciência e Tecnologia de Alimentos, v. 28, n. 2, abr./jun. 2008, p. 263-268.

SANTOS, G. M.; MAIA, G. A.; SOUSA, P. H. M.; FIGUEIREDO, R. W.; COSTA, J. M.C.;

FONSECA, A.V.V. Atividade Antioxidante e Correlações com Componentes Bioativas de Produtos Comerciais de Cupuaçu. Ciência Rural, Santa Maria, Online. Fortaleza CE, 2010. ISSN 0103-8478.

SANTOS, K. A.; FAIX, P. N.; SANTOS, E. F. dos. MANHANI, M. R.; SILVA, E. C. da. NOVELLO, D. Efeito da adição de inulina em geleia de abacaxi: análise físico-química e sensorial entre escolares. O Mundo da Saúde, São Paulo - 2014;38(3):286-295

SEGOVIA, J.F.O. Dimensão da agricultura familiar e das cadeias mercantis envolvidas na comercialização da produção no espaço amapaense. Tese (doutorado)Universidade Federal do Pará, Núcleo de Altos Estudos Amazônicos, Programa de Pós-Graduação em Desenvolvimento Sustentável do Trópico Úmido, Belém, 2011. 343p.

SILVA, N. da; JUNQUEIRA, V. C. A.; SILVEIRA, N. F. A. Manual de métodos de análise microbiológica de alimentos. 2. ed. São Paulo: Livraria Varela, 2001

SILVA, H. M. da. Caracterização físico-química e informações nutricionais de doce em massa de cupuaçu. Imperatriz, MA, 2014.

DA SILVA, A. S. S.; FARIAS, L. F. Elaboração da farinha à base da amêndoa do cupuaçu Theobroma grandiflorum Schum. Revista Arquivos Científicos (IMMES), v. 1, n. 1, p. 36-42, 2018.

SOUZA, J. M.L. de. Geleia de cupuaçu / Joana Maria Leite de Souza, Fabiana Silva Reis, Felícia Maria Nogueira Leite, Dorila Silva de Oliveira Mota Gonzaga. - Brasília, DF: Embrapa Informação Tecnológica, 2007. 47 p.

SUCUPIRA, N.R.; SILVA, A.B.; PEREIRA, G.; COSTA, J.N. Métodos Para Determinação da Atividade Antioxidante de Frutos. UNOPAR Cient. Ciênc. Biol. Saúde. Fortaleza CE, 2012;14(4):263-9.

Submissão: 08/09/2020

Aprovado para publicação: 22/11/2020 